\title{
Tight Error Bounds for Space-Time Orthogonal Block Codes Under Slow Rayleigh Flat Fading
}

\author{
Firouz Behnamfar, Member, IEEE, Fady Alajaji, Senior Member, IEEE, and Tamás Linder, Senior Member, IEEE
}

\begin{abstract}
The performance of space-time orthogonal block (STOB) codes over slow Rayleigh fading channels and maximum-likelihood (ML) decoding is investigated. Two Bonferroni-type bounds (one upper bound and one lower bound) for the symbol error rate (SER) and bit error rate (BER) of the system are obtained. The bounds are expressed in terms of the pairwise error probabilities (PEPs) and the two-dimensional pairwise error probabilities (2-D PEPs) of the transmitted symbols. Furthermore, the bounds can be efficiently evaluated and they hold for arbitrary (nonstandard) signaling schemes and mappings. Numerical results demonstrate that the bounds are very accurate in estimating the performance of STOB codes. In particular, the upper and lower bounds often coincide even at low channel signal-to-noise ratios, large constellation sizes, and large diversity orders.
\end{abstract}

Index Terms-Bit and symbol error rates, diversity, maximumlikelihood decoding, multiple antennas, pairwise error probability, slow Rayleigh fading, space-time coding, wireless communications.

\section{INTRODUCTION}

$\mathbf{T}$ THE original papers on space-time trellis (STT) codes [15] and space-time orthogonal block codes [14] adopt the Chernoff upper bound to estimate the pairwise error probability (PEP) of codewords and to establish code design criteria. Although the Chernoff bound yields successful code constructions, it is quite loose even at high values of channel signal-to-noise ratio (CSNR). Furthermore, it is common practice to use the union bound to approximate the symbol error rate or bit error rate. However, the union bound is intrinsically loose, particularly at low CSNRs. Therefore, using the Chernoff bound together with the union bound may result in poor approximations to system performance (see, e.g., Section IV).

The main challenge in determining the codeword PEP of space-time codes under maximum-likelihood detection is to average the Gaussian $Q$-function $Q(X)$, where $X$ is a nonnegative random variable. This problem has been mainly studied in [11], [13], and [16]. In [16], a method to derive the exact PEP in terms of the residues of the moment generating function (MGF) is presented. The result is used to find an estimate of the BER of STT codes. In [13] a form of the MGF is derived which

Paper approved by H. El-Gamal, the Editor for Space-Time Coding and Spread Spectrum of the IEEE Communications Society. Manuscript received July 22, 2003; revised June 9, 2004. This work was supported in part by the Natural Sciences and Engineering Research Council (NSERC) of Canada and the Premier's Research Excellence Award (PREA) of Ontario. This paper was presented in part at the 2003 IEEE International Symposium on Information Theory (ISIT'03), Yokohama, Japan, June-July 2003.

F. Behnamfar is with the Department of Mathematics and Statistics, Queen's University, Kingston, ON K7L 3N6, Canada (e-mail: firouz@mast.queensu.ca).

F. Alajaji and T. Linder are with the Department of Mathematics and Statistics and the Department of Electrical and Computer Engineering, Queen's University, Kingston, ON K7L 3N6, Canada (e-mail: fady@mast.queensu.ca; linder@mast.queensu.ca).

Digital Object Identifier 10.1109/TCOMM.2005.849797 is simpler to evaluate for block fading channels, and the PEPs are found numerically. The study in [11] presents another exact form of the PEP, which can be evaluated in closed form when the codewords corresponding to a pair of trellis paths have orthogonal rows. Recently, Lu et al. derived exact closed-form expressions for the PEP of general space-time codes [7]. Their derivation of the STOB codeword PEP is based on averaging Craig's formula [5] using the results in [12].

With respect to the SER/BER analysis of space-time orthogonal block codes, one can use the closed-form conditional SER formulas in [12] and average them over the fading coefficients distribution to derive exact SER formulas for standard PSK and square $2^{2 p}$-ary QAM, where $p$ is a positive integer; this approach is taken in [9]. However, the resulting SER formulas do not hold for arbitrary constellations such as star-QAM and it is not clear how to extend this approach to evaluate the BER, specially if Gray mapping is not used. For PSK signaling, tight bounds on the symbol PEP were found in [4]. Also, in [1], the symbol PEP was found in integral form for diversity reception systems, which can be easily tailored to STOB coding. The symbol PEPs can be used to derive an upper bound on the BER; however, as will be shown in the sequel, the resulting BER upper bound is very loose even at medium CSNRs.

In this work, we show how to use the symbol PEP results to derive bounds for the SER and BER of STOB codes via establishing two algorithmic Bonferroni-type upper and lower bounds. The bounds are general (upper/lower) bounds on the probability of a union of a finite number of events $P\left(\cup_{i=1}^{n} A_{i}\right)$ which are expressed in terms of the individual probabilities $P\left(A_{i}\right), i=1, \ldots, n$ as well as the probabilities of intersections of pairs of events $P\left(A_{i} \cap A_{j}\right), i, j=1, \ldots, n, i<j$ [6]. When specialized to the STOB codes setting, the overall error event of the system can be represented by such a finite union of events, where $P\left(A_{i}\right)$ is the PEP, and $P\left(A_{i} \cap A_{j}\right)$ is the two-dimensional (2-D) pairwise error probability which we determine in closed form. One important feature of the bounds is that they hold for arbitrary constellations and signal mappings since they do not depend on the geometry of the system at hand. Numerical results indicate that the bounds for STOB codes often coincide with the true error probabilities obtained via simulations even at low CSNRs. Furthermore, the computational complexity of these algorithmic bounds is very modest even for large constellations and larger number of antennas.

The rest of this letter is organized as follows. The channel model and a review of space-time orthogonal block codes are presented in Section II. Section III briefly describes the algorithmic lower and upper bounds of [6] and derives the exact probability expressions needed for applying the bounds to STOB coding systems. Numerical and simulation results are given in Section IV. Section V concludes the paper. 


\section{Symbol Pairwise ERror Probabilities of SPACE-Time ORTHOGONAL CODES}

The multiantenna communication system considered here employs $K$ transmit and $L$ receive antennas. The input to the system is an independent and identically distributed (i.i.d.) uniform bit-stream. The complex baseband constellation points are denoted by $\left\{c_{k}\right\}_{k=1}^{2^{p}}$ where $p$ is a positive integer. We assume that the signal constellation has an average energy of one. In order to have an average CSNR of $\gamma_{s}$ at each receive antenna, the modulators weight the symbols by $\sqrt{\gamma_{s} / K}$ (see (1)). The channel is assumed to be Rayleigh flat fading, so that the complex path gain from transmit antenna $i$ to receive antenna $j, H_{j i}$, has a zero-mean unit-variance complex Gaussian distribution, denoted by $\mathcal{C N}(0,1)$, with i.i.d. real and imaginary parts. We assume that the receiver, but not the transmitter, has perfect knowledge of the path gains. Moreover, we assume that the channel is quasi-static, meaning that the path gains remain constant during the transmission of a codeword. The additive noise at receiver $j$ at symbol interval $t$, $N_{t}^{j}$, is assumed to be $\mathcal{C N}(0,1)$ distributed with i.i.d. real and imaginary parts. The signal at receive antenna $j$ can be written as $R_{t}^{j}=\sqrt{\gamma_{s} / K} \sum_{i=1}^{K} H_{j i} s_{t}^{i}+N_{t}^{j}$, where $\sqrt{\gamma_{s} / K} s_{t}^{i}$ is the signal sent from antenna $i$, or in matrix form

$$
\boldsymbol{r}_{t}=\sqrt{\frac{\gamma_{s}}{K}} \boldsymbol{H} \mathbf{s}_{t}+\boldsymbol{n}_{t}
$$

where $\boldsymbol{r}_{t}=\left(R_{t}^{1}, \ldots, R_{t}^{L}\right)^{T}, \mathbf{s}_{t}=\left(s_{t}^{1}, \ldots, s_{t}^{K}\right)^{T}$, $\boldsymbol{n}_{t}=\left(N_{t}^{1}, \ldots, N_{t}^{L}\right)^{T}$, and ${ }^{T}$ denotes transposition. $\boldsymbol{H}$ is the $L \times K$ path gains matrix with elements $\left\{H_{j i}\right\}$. We assume that the input, noise, and fading processes are all independent.

Let $\mathbf{c}=\left(c_{1}, \ldots, c_{\tau}\right)^{T}$ be a vector of $\tau$ consecutive constellation points and $\mathbf{S}=\left(\mathbf{s}_{1}, \ldots, \mathbf{s}_{T_{w}}\right)$ be the space-time code corresponding to it, where $T_{w}$ is the codeword length. In the case of STOB codes, we have $T_{w}=g \tau$, where $g$ is the coding gain and $\mathbf{S} \mathbf{S}^{\dagger}=g\|\mathbf{c}\|^{2} / \mathbf{I}_{\tau}$, where $\boldsymbol{I}_{\tau}$ is the $\tau \times \tau$ identity matrix and $\dagger$ represents complex conjugate transposition. As an example, for the code $\mathcal{G}^{3}$ in [14], $T_{w}=8, \tau=4$, and $g=2$, and for Alamouti's code [2], $g=1$ and $T_{w}=\tau=2$.

It can be verified that the PEP conditioned on the path gains is given by

$$
P\left(c_{i} \rightarrow c_{j} \mid \boldsymbol{H}\right)=Q\left(\delta_{i j} \sqrt{Y}\right)
$$

where $c_{i} \rightarrow c_{j}$ denotes the event that $c_{j}$ has a larger metric than $c_{i}$ when $c_{i}$ is sent $\left(c_{i}\right.$ and $c_{j}$ are a pair of symbols input to the STOB encoder), $\delta_{i j}=\sqrt{g \gamma_{s} / 2 K}\left|c_{i}-c_{j}\right|$, and

$$
Y=\sum_{l=1}^{L} Y_{l}=\sum_{k=1}^{K} \sum_{l=1}^{L}\left|H_{l k}\right|^{2}
$$

is the sum of the squared magnitudes of all path gains. ${ }^{1}$ Using the moment generating function of Gaussian random

${ }^{1}$ We assume here that all path gains contribute equally in $Y$. This assumption is valid for most orthogonal codes used in the literature, including Alamouti's $\mathcal{G}^{2}$ code and the $\mathcal{G}^{3}$ and $\mathcal{G}^{4}$ codes of [14]. variables, it can be verified that the probability density function of $Y$ is

$$
f_{Y}(y)=\frac{1}{(n-1) !} y^{n-1} e^{-y}, \quad y>0
$$

where $n=K L$. As noticed in [3], the PEP of diversity reception systems with maximum ratio combining has a form similar to that of (2) with the exception that $\delta_{i j}$ is given by $\sqrt{\gamma_{s} / 2}\left|c_{i}-c_{j}\right|$. Therefore, the conditional PEP for those systems is the same as (2). MRC systems were previously analyzed in, for example, [8], [12], whose results can be used to obtain the expected value of (2) as

$$
P\left(c_{i} \rightarrow c_{j}\right)=\frac{1}{2}\left(1-\frac{\delta_{i j}}{\sqrt{\delta_{i j}^{2}+2}} \sum_{k=0}^{K L-1}\left(\begin{array}{c}
2 k \\
k
\end{array}\right) \frac{1}{\left(2\left(\delta_{i j}^{2}+2\right)\right)^{k}}\right) .
$$

\section{ERROR RATE BOUNDS FOR SPACE-TIME ORTHOGONAL BLOCK CODES}

In this section, we show how the algorithms in [6] for two tight Bonferroni-type upper and lower bounds can be used for space-time orthogonal block codes.

\section{A. The Bonferroni-Type Lower and Upper Bounds}

For a positive integer $M$, let $A_{1}, \ldots, A_{M}$ be events in an arbitrary probability space. A stepwise algorithm is given in [6] to find a lower bound for the probability of the union of the $A_{i}$. The bound is given by

$$
P\left(\bigcup_{i=1}^{M} A_{i}\right) \geq \max _{\mathcal{I}}\left\{\sum_{i \in \mathcal{I}} P\left(A_{i}\right)-\sum_{\substack{i, j \in \mathcal{I} \\ i<j}} P\left(A_{i} \cap A_{j}\right)\right\}
$$

where $\mathcal{I} \subseteq\{1,2, \ldots, M\}$. Also, a greedy algorithm is described in [6] which finds an upper bound for the probability of the union of $A_{i}$, which is given by

$$
P\left(\bigcup_{i=1}^{M} A_{i}\right) \leq \sum_{i=1}^{M} P\left(A_{i}\right)-\max _{T_{0} \in \mathcal{T}} \sum_{(i, j) \in T_{0}} P\left(A_{i} \cap A_{j}\right)
$$

where $\mathcal{T}$ is the set of all spanning trees of the $M$ indices, i.e., the trees whose set of nodes is the set of indexes $\{1, \ldots, M\}$. The greedy algorithm in [6] is as follows. First, each pair of nodes $(i, j)$ is connected via edges of weight $P\left(A_{i} \cap A_{j}\right)$ to form a fully connected graph. To form the tree $T_{0}$, the algorithm starts from the edge with the largest weight (the $(i, j)$ with the largest $P\left(A_{i} \cap A_{j}\right)$ ). Then, at each step, the edge with the largest weight is added to $T_{0}$, subject to the constraint that there is no cycle in $T_{0}$. This step is executed until all of the nodes are in $T_{0}$.

\section{B. Symbol Error Rate}

For a constellation of size $M=2^{p}$, where $p$ is a positive integer, and an i.i.d. uniform bit stream, the SER is given by

$$
\mathrm{SER}=\sum_{u=1}^{M} P\left(\epsilon \mid c_{u}\right) P\left(c_{u}\right)=\frac{1}{M} \sum_{u=1}^{M} P_{u}\left(\bigcup_{i \neq u} \epsilon_{i u}\right)
$$


where $P\left(\cdot \mid c_{u}\right) \triangleq P_{u}(\cdot)$ is the conditional probability of error given that $c_{u}$ was sent, and $\epsilon_{i u}$ indicates the event that $c_{i}$ has a larger metric than $c_{u}$. Note that $P_{u}\left(\epsilon_{i u}\right)$ is the PEP and is given in (5). In order to find lower and upper bounds on the probability of each union in (8) via (6) and (7), respectively, we need to find the probability of the intersection of $\epsilon_{i u}$ and $\epsilon_{j u}$, the 2-D PEP of symbols $c_{i}$ and $c_{j}$ with $c_{u}$. This can be done by averaging [6, eq. (7)], i.e.,

$$
P_{u}\left(\epsilon_{i u} \cap \epsilon_{j u}\right)=\mathrm{E}_{Y}\left\{\Psi\left(\rho_{i j u}, \delta_{i u} \sqrt{Y}, \delta_{j u} \sqrt{Y}\right)\right\}
$$

where $\rho_{i j u}=\left\langle c_{i}-c_{u}, c_{j}-c_{u}\right\rangle / d_{i u} d_{j u},\langle x, y\rangle=$ $\Re\{x\} \Re\{y\}+\Im\{x\} \Im\{y\}(\Re\{\cdot\}$ and $\Im\{\cdot\}$ are the real and imaginary parts, respectively), $d_{i u}=\left|c_{i}-c_{u}\right|, Y$ is defined in (3), and

$$
\begin{aligned}
& \Psi\left(\rho, \phi_{i}(y), \phi_{j}(y)\right) \\
& =\frac{1}{2 \pi \sqrt{1-\rho^{2}}} \int_{\phi_{i}(y)}^{\infty} \int_{\phi_{j}(y)}^{\infty} \exp \left(-\frac{\tau^{2}-2 \rho \tau \lambda+\lambda^{2}}{2\left(1-\rho^{2}\right)}\right) d \tau d \lambda
\end{aligned}
$$

with $\phi_{i}(y)=\delta_{i u} \sqrt{y}$. As $\phi_{i}(y)$ and $\phi_{j}(y)$ are nonnegative, we can use the result of [10] to write (10) as

$$
\begin{array}{r}
\Psi\left(\rho, \delta_{i u} \sqrt{y}, \delta_{j u} \sqrt{y}\right)=\frac{1}{2 \pi} \int_{0}^{\varphi\left(d_{i u} / d_{j u}, \rho\right)} \exp \left(\frac{-\delta_{i u}^{2}}{2 \sin ^{2} \theta} y\right) d \theta \\
+\frac{1}{2 \pi} \int_{0}^{\varphi\left(d_{j u} / d_{i u}, \rho\right)} \exp \left(\frac{-\delta_{j u}^{2}}{2 \sin ^{2} \theta} y\right) d \theta
\end{array}
$$

where $\varphi(x, \rho)=\tan ^{-1}\left(x \sqrt{1-\rho^{2}} /(1-\rho x)\right), \operatorname{and}^{-1}(x)$ is defined here as $\pi-\tan ^{-1}(-x)$ for negative $x$. Using the pdf of $Y$ in (4), we find the expected value of each of the integrals in (11) as follows:

$$
\begin{aligned}
& E_{Y}\left[\int_{0}^{\varphi} e^{-a(\theta) Y} d \theta\right] \\
& =\frac{1}{(n-1) !} \int_{0}^{\infty} y^{n-1} e^{-y} \int_{0}^{\varphi} e^{-a(\theta) y} d \theta d y \\
& =\frac{1}{(n-1) !} \int_{0}^{\varphi} \int_{0}^{\infty} y^{n-1} e^{-(1+a(\theta)) y} d y d \theta \\
& =\int_{0}^{\varphi}\left(\frac{1}{1+a(\theta)}\right)^{n} d \theta
\end{aligned}
$$

where $a(\theta)$ is a given nonnegative function of $\theta$. The step from (12) to (13) follows (for example) by writing the inner integral in (12) in terms of the $(n-1)^{\text {st }}$ derivative of the Laplace transform of the unit step function. Using (13) with $a(\theta)=\delta^{2} / 2 \sin ^{2} \theta$ and [12, eq. 5A.35], we have

$$
\begin{aligned}
& I(\varphi, \delta) \\
& \triangleq \frac{1}{2 \pi} \int_{0}^{\varphi}\left(\frac{2 \sin ^{2} \theta}{\delta^{2}+2 \sin ^{2} \theta}\right)^{n} d \theta \\
& =\frac{\varphi}{2 \pi}-\frac{\beta}{2 \pi} \frac{\delta}{\sqrt{2+\delta^{2}}} \sum_{k=0}^{n-1}\left(\begin{array}{c}
2 k \\
k
\end{array}\right) \frac{1}{\left(2\left(2+\delta^{2}\right)\right)^{k}} \\
& -\frac{\delta}{\pi \sqrt{2+\delta^{2}}} \sum_{k=0}^{n-1} \sum_{m=0}^{k-1}\left(\begin{array}{c}
2 k \\
m
\end{array}\right) \frac{(-1)^{m+k}}{\left(2\left(2+\delta^{2}\right)\right)^{k}} \frac{\sin [2 \beta(k-m)]}{2(k-m)}
\end{aligned}
$$

where

$$
\beta=\frac{1}{2} \tan ^{-1} \frac{A}{B}+\frac{\pi}{2}\left[1-\left(\frac{1+\operatorname{sgn} B}{2}\right) \operatorname{sgn} A\right]
$$

with $A=\delta \sqrt{2+\delta^{2}} \sin 2 \varphi, B=\left(1+\delta^{2}\right) \cos 2 \varphi-1$, and $\operatorname{sgn} x \triangleq|x| / x$ if $x \neq 0$ and 0 otherwise. Therefore, from (9), (11), and (14), we obtain the following expression for the 2-D PEP:

$$
P_{u}\left(\epsilon_{i u} \cap \epsilon_{j u}\right)=I\left(\varphi\left(\frac{\delta_{i u}}{\delta_{j u}}, \rho_{i j u}\right), \delta_{i u}\right)+I\left(\varphi\left(\frac{\delta_{j u}}{\delta_{i u}}, \rho_{i j u}\right), \delta_{j u}\right)
$$

\section{Bit Error Rate}

The same algorithm can be used as outlined in [6] to estimate the BER. We have

$$
\mathrm{BER}=\frac{1}{M} \sum_{u=1}^{M} P_{b}(u),
$$

where $P_{b}(u)$ is the bit error probability when $c_{u}$ is sent and is given by

$$
\begin{aligned}
P_{b}(u) & =\frac{1}{\log _{2} M} \sum_{j=1}^{M} H(j, u) P\left(\hat{c}=c_{j} \mid c=c_{u}\right) \\
& =\frac{1}{\log _{2} M} \sum_{j=1}^{M} H(j, u)\left(1-P_{u}\left(\bigcup_{i \neq j} \epsilon_{i j}\right)\right)
\end{aligned}
$$

where $H(j, u)$ is the Hamming distance between the bit assignments of $c_{j}$ and $c_{u}, P\left(\hat{c}=c_{j} \mid c=c_{u}\right)$ is the probability that $c_{j}$ is decoded given that $c_{u}$ is sent, and $\epsilon_{i j}$ is the event that $c_{i}$ has a larger metric than $c_{j}$. From the above, it is clear that finding upper and lower bounds on the BER requires evaluating lower and upper bounds on the probability of the union in (16). These bounds, in turn, require the computation of

$$
\begin{aligned}
P_{u}\left(\epsilon_{i j}\right)= & E_{Y}\left\{Q\left(\frac{\delta_{i u}^{2}-\delta_{j u}^{2}}{\delta_{i j}} \sqrt{Y}\right)\right\} \\
= & \frac{1}{2}\left(1-\sqrt{\frac{\delta_{i u}^{2}-\delta_{j u}^{2}}{\left(\delta_{i u}^{2}-\delta_{j u}^{2}\right)^{2}-2 \delta_{i j}^{2}}}\right) \\
& \times \sum_{k=0}^{K L-1}\left(\begin{array}{c}
2 k \\
k
\end{array}\right)\left(\frac{\delta_{i j}^{2}}{2\left(\left(\delta_{i u}^{2}-\delta_{j u}^{2}\right)^{2}+2 \delta_{i j}^{2}\right)}\right)^{k}
\end{aligned}
$$

as well as

$$
\begin{aligned}
P_{u}\left(\epsilon_{i j} \cap \epsilon_{k j}\right)= & E_{Y}\left\{\Psi\left(\rho_{i k j}, \frac{\delta_{i u}^{2}-\delta_{j u}^{2}}{\delta_{i j}} \sqrt{Y}, \frac{\delta_{k u}^{2}-\delta_{j u}^{2}}{\delta_{k j}} \sqrt{Y}\right)\right\} \\
= & I\left(\varphi\left(\frac{\delta_{k j}\left(\delta_{i u}^{2}-\delta_{j u}^{2}\right)}{\delta_{i j}\left(\delta_{k u}^{2}-\delta_{j u}^{2}\right)}, \rho_{i k j}\right), \frac{\delta_{i u}^{2}-\delta_{j u}^{2}}{\delta_{i j}}\right) \\
& +I\left(\varphi\left(\frac{\delta_{i j}\left(\delta_{k u}^{2}-\delta_{j u}^{2}\right)}{\delta_{k j}\left(\delta_{i u}^{2}-\delta_{j u}^{2}\right)}, \rho_{i k j}\right), \frac{\delta_{k u}^{2}-\delta_{j u}^{2}}{\delta_{k j}}\right)
\end{aligned}
$$

where $I(\cdot, \cdot)$ is defined in (14) and if the second and the third arguments of $\Psi(\cdot, \cdot, \cdot)$ are nonnegative. If at least one of the two 


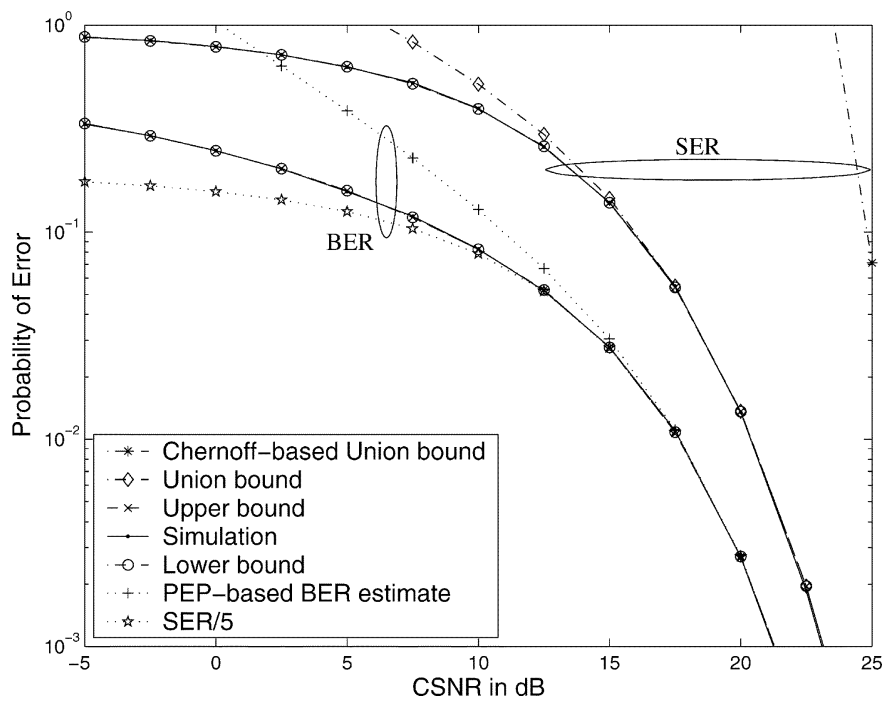

Fig. 1. Results for 32-PSK signaling with $K=2, L=4$, and $\mathcal{G}^{2}$ STOB code $(5 \mathrm{bits} / \mathrm{s} / \mathrm{Hz})$

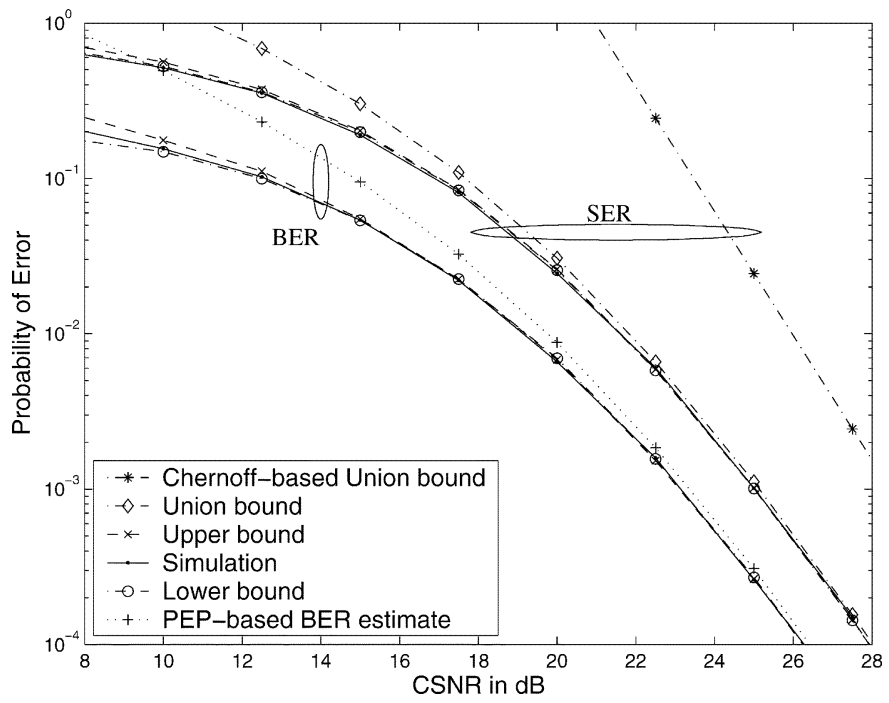

Fig. 2. Results for 64-QAM signaling with $K=2, L=2$, and $\mathcal{G}^{2}$ STOB code $(6 \mathrm{bits} / \mathrm{s} / \mathrm{Hz})$.

arguments are negative, the $\Psi(\cdot, \cdot, \cdot)$ function can be written as sum of a number of $\Psi(\cdot, \cdot, \cdot)$ functions with nonnegative arguments as shown in the equation at the bottom of the page. In this case, we can also obtain an expression for the 2-D PEP as shown in (17).

\section{Simulation Results}

For this part, the length of the input bit-sequence is $\max \left(100000,100 \times \mathrm{BER}^{-1}\right)$. The computation time of the algorithmic Bonferroni bounds is negligible for our code

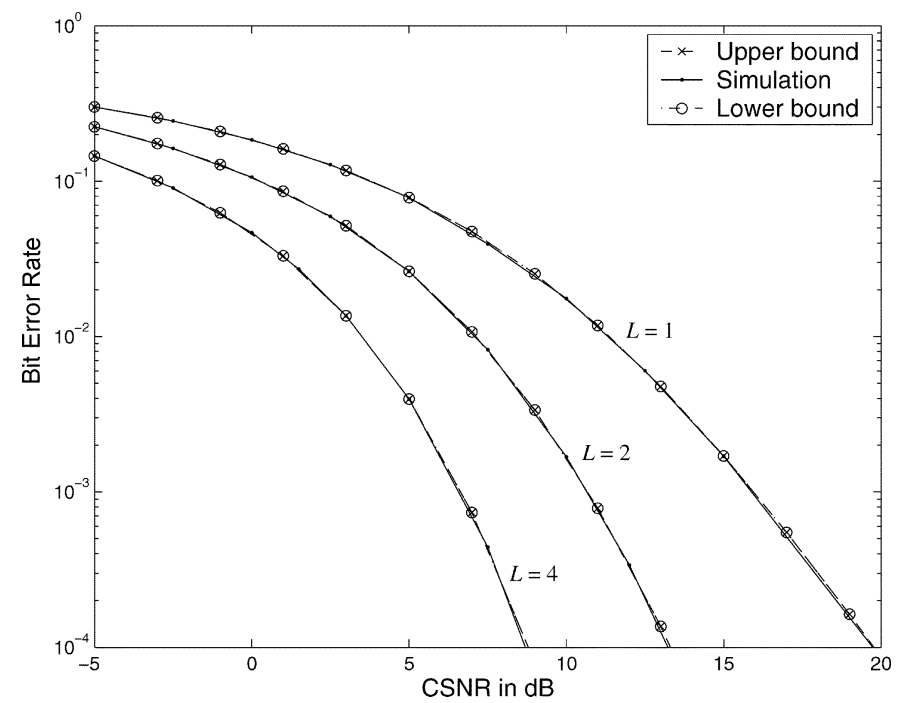

Fig. 3. Results for 8-PSK signaling with $K=3, L=1,2$, and 4, and $\mathcal{G}^{3}$ STOB code $(1.5 \mathrm{bits} / \mathrm{s} / \mathrm{Hz})$.

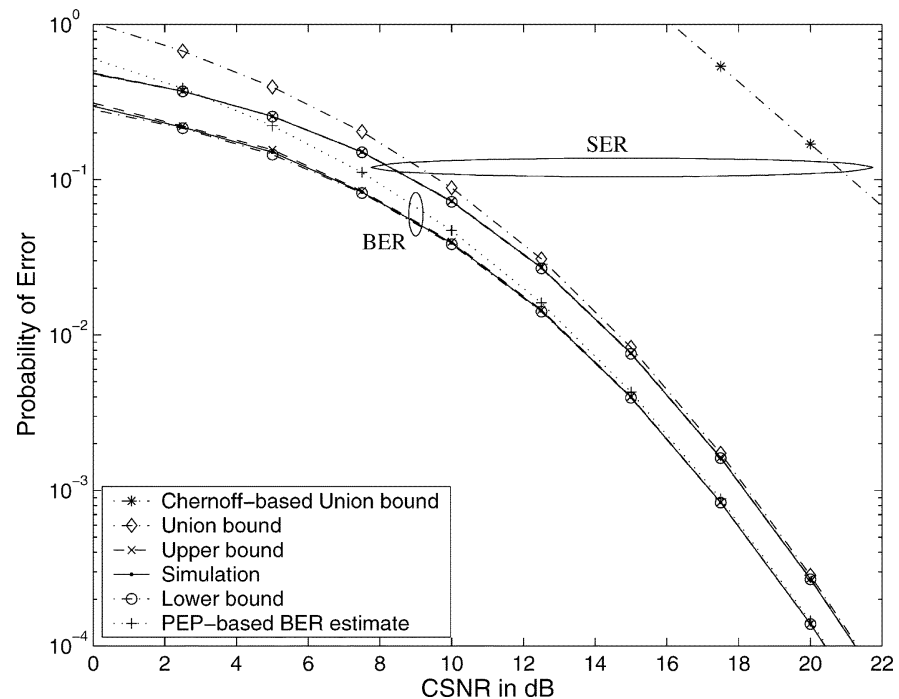

Fig. 4. Results for Star 8-QAM signaling and quasi-Gray mapping with $K=$ $4, L=1$, and $\mathcal{G}^{4}$ STOB code $(1.5 \mathrm{bits} / \mathrm{s} / \mathrm{Hz})$.

that is written in C and run on a SUN Ultra 60 machine. The Chernoff and the union bounds are also given for the SER.

The SER and BER versus CSNR curves are presented in Figs. 1-4 for various $K, L$, space-time codes, and $M$-ary PSK and QAM constellations with Gray or quasi-Gray mappings. Figs. 1 and 2 show the performance of Alamouti's $\mathcal{G}^{2}$ code [2], while the performance of the codes $\mathcal{G}^{3}$ and $\mathcal{G}^{4}$ of [14] are presented in Figs. 3 and 4, respectively. The lower and upper Bonferroni bounds based on the 2-D PEP formulas for both SER and BER curves are very tight and can hardly be distinguished from

$$
\Psi(\rho, \alpha, \beta)= \begin{cases}\Psi(\rho, \alpha, 0)+\Psi(-\rho, \alpha, 0)-\Psi(-\rho, \alpha,-\beta) & \alpha \geq 0, \beta<0 \\ \Psi(\rho, 0, \beta)+\Psi(-\rho, 0, \beta)-\Psi(-\rho,-\alpha, \beta) & \alpha<0, \beta \geq 0 \\ 1-\Psi(\rho, 0,-\beta)-\Psi(-\rho, 0,-\beta)-\Psi(\rho,-\alpha, 0)-\Psi(-\rho,-\alpha, 0)+\Psi(\rho,-\alpha,-\beta) & \alpha<0, \beta<0\end{cases}
$$


each other and from the performance curve obtained via simulation. It can be seen that as the constellation size grows, the union bound becomes less reliable and the Chernoff bound gets farther from the other curves, while the lower and upper bounds based on the exact 2-D PEP remain very tight. Fig. 3 shows our BER upper and lower bounds as well as simulation results for a system with three transmit and $L=1,2$, and 4 receive antennas for a wide range of CSNRs. The bounds are tight even at negative CSNR values and high diversity orders. Notice that the Star 8-QAM constellation of Fig. 4 (with signal points at $(1 / 2 \sqrt{10})\{1,3, i, 3 i,-1,-3,-i,-3 i\}$, where $i=\sqrt{-1}$ and a corresponding quasi-Gray bit mapping given by $\{000,100$, $001,101,011,111,010,110\})$ is not a regular square QAM nor 8-PSK, but the bounds are still very tight.

An upper bound on the BER can be computed by upper bounding $P\left(\hat{c}=c_{j} \mid c=c_{u}\right)$ in (16) by the PEP to get

$$
\mathrm{BER} \leq \frac{1}{M \log _{2} M} \sum_{u} \sum_{j} H(u, j) P\left(c_{u} \rightarrow c_{j}\right),
$$

where $H(u, j)$ is the Hamming distance between the bits corresponding to $c_{u}$ and $c_{j}$. This bound is also plotted in all figures. It is clearly observed that our upper bound is much tighter, particularly for larger constellations.

As Gray mapping is used in the above systems (except for Fig. 4), it is expected that at high enough CSNRs the BER of PSK-modulated systems converges to [8] BER $\approx(1 / p) \mathrm{SER}$, where $2^{p}$ is the constellation size. The curves labeled with "SER/p" in Fig. 1 show this approximation. We also observe that as the constellation size shrinks or the number of the receive antennas grows, this estimate becomes tighter. Therefore, at high enough CSNR values, it would be enough to find only one of the SER bounds to obtain a good estimate of system SER and BER. For example, for 16-PSK signaling and at a CSNR of $15 \mathrm{~dB}$, the coinciding SER lower and upper bounds are equal to 0.195682 . The above approximation yields BER $\approx 0.048921$, while the lower and upper bounds on the BER coincide and equal 0.051838 . It is also important to note that although we have only presented the BER results for the Gray or quasi-Gray signal mappings, our bounds also apply to any other mapping and show the same behavior.

\section{CONCLUSION}

We derive very tight Bonferroni-type lower and upper bounds on the symbol and bit error rates for space-time orthogonal block codes with arbitrary signal constellations. The lower and upper bounds derived for these codes have very close values (up to six identical significant digits) even at low channel signal-to-noise ratios. Several constellation types and sizes, space-time codes, and diversity orders were considered in the numerical results. Simulations support the tightness and accuracy of the formulas derived.

\section{ACKNOWLEDGMENT}

The authors would like to thank Dr. M. K. Simon for his constructive comments and for bringing (14) to their attention.

\section{REFERENCES}

[1] I. S. Abdel-Ghaffar and S. Pasupathy, "Asymptotical performance of $M$-ary and binary signals over multipath/multichannel Rayleigh and Rician fading," IEEE Trans. Commun., vol. 43, no. 11, pp. 2721-2731, Nov. 1995.

[2] S. M. Alamouti, "A simple transmit diversity technique for wireless communications," J. Sel. Areas Commun., vol. 16, no. 8, pp. 1451-1458, Oct. 1998.

[3] G. Bauch, J. Hagenauer, and N. Seshadri, "Turbo processing in transmit antenna diversity systems," Ann. Telecommun., vol. 56, pp. 455-471, Aug. 2001

[4] A. Conti, M. Win, and M. Chiani, "On the inverse symbol-error probability for diversity reception," IEEE Trans. Commun., vol. 51, no. 5, pp. 753-756, May 2003.

[5] J. W. Craig, "A new, simple, and exact result for calculating the probability of error for two-dimensional signal constellations," in Proc. IEEE Military Commun. Conf., McLean, VA, Nov. 1991, pp. 571-575.

[6] H. Kuai, F. Alajaji, and G. Takahara, "Tight error bounds for nonuniform signaling over AWGN channels," IEEE Trans. Inf. Theory, vol. 46, no. 11, pp. 2712-2718, Nov. 2000.

[7] H. Lu, Y. Wang, P. V. Kumar, and K. Chugg, "Remarks on space-time codes including a new lower bound and an improved code," IEEE Trans. Inf. Theory, vol. 49, no. 10, pp. 2752-2757, Oct. 2003.

[8] J. G. Proakis, Digital Communications, 4th ed. New York: McGrawHill, 2001

[9] H. Shin and J. H. Lee, "Exact symbol error probability of orthogonal space-time block codes," in Proc. IEEE Global Commun. Conf., vol. 2, Nov. 2002, pp. 1197-1201.

[10] M. K. Simon, "A simpler form of the Craig representation for the twodimensional joint Gaussian Q-function," IEEE Commun. Lett., vol. 6, no. 2, pp. 49-51, Feb. 2002.

[11] - "Evaluation of average bit error probability for space-time coding based on a simpler exact evaluation of pairwise error probability," $J$. Commun. Netw., vol. 3, pp. 257-264, Sep. 2001.

[12] M. K. Simon and M. S. Alouini, Digital Communications over Fading Channels: A Unified Approach to Performance Analysis. New York: Wiley, 2000.

[13] G. Taricco and E. Biglieri, "Exact pairwise error probability of spacetime codes," IEEE Trans. Inf. Theory, vol. 48, no. 2, pp. 510-513, Feb. 2002.

[14] V. Tarokh, H. Jafarkhani, and A. R. Calderbank, "Space-time block codes from orthogonal designs," IEEE Trans. Inf. Theory, vol. 45, no. 7, pp. 1456-1467, Jul. 1999.

[15] V. Tarokh, N. Seshadri, and A. R. Calderbank, "Space-time codes for high data rate wireless communication: Performance criteria and code construction," IEEE Trans. Inf. Theory, vol. 44, no. 3, pp. 744-765, Mar. 1998.

[16] M. Uysal and C. N. Georghiades, "Error performance analysis of spacetime codes over Rayleigh fading channels," J. Commun. Netw., vol. 2, pp. 351-356, Dec. 2000 